\title{
Is Otilonium Bromide Really Effective for Treating Asian Patients With Irritable Bowel Syndrome?
}

TO THE EDITOR: Irritable bowel syndrome (IBS) is a complex and very common disease. It is a condition characterized by abdominal pain/discomfort associated with disordered defecation in the absence of any organic abnormalities. Although the exact pathophysiology of IBS is not fully understood, the treatment of IBS is targeted at the management of abdominal pain/discomfort including pharmacotherapy with antispasmodics. ${ }^{1}$ Mebeverine is a musculotropic agent that has antispasmodic activity and regulatory effects on the bowel functions, and has been effectively used in the treatment of IBS for many years. ${ }^{2}$ Otilonium bromide $(\mathrm{OB})$ is a quaternary ammonium compound whose spasmolytic action is mainly due to its direct myolytic properties. ${ }^{3}$ In the meta-anlaysis of smooth muscle relaxants in the treatment of IBS, both mebeverine and $\mathrm{OB}$ were superior to placebo in relieving symptoms such as abdominal pain and distension without adverse effects. ${ }^{4}$ In addition, a double-blind, placebo-controlled study among Italian IBS patients showed that the reduced episode of abdominal pain was higher in OB treated patients (53.3\%) than in placebo patients (39.9\%). ${ }^{5}$

With great interest, I read the report by Chang et al ${ }^{6}$ regarding "The evaluation of otilonium bromide treatment in Asian patients with IBS." In this study, overall 117 IBS patients meeting Rome II criteria were enrolled in an 8-week trial, and randomized participants received either OB $40 \mathrm{mg}$ or mebeverine 100 mg 3 doses daily. The primary endpoints were to evaluate the changes of abdominal pain/discomfort frequency scores (APDFS) and safety profile. The authors showed that the reduced APDFS in $\mathrm{OB}$ and mebeverine were $0.55 \pm 1.20(P=0.011)$ and 0.37 $\pm 1.11(P=0.042)$, respectively, after 8 -week treatment compared to baseline. The most reported side effects were dry mouth, nausea and dizziness. OB was not inferior to mebeverine in treating IBS patients with alleviations in abdominal pain intensity, flatulence and abdominal bloating. These results suggest that $\mathrm{OB}$ is as effective as mebeverine for alleviating IBS symptoms in Oriental populations.
Although the authors showed an important result of $\mathrm{OB}$ treatment for Asian IBS patients, there are a few limitations regarding this study. First, the dose of mebeverine used was 400 $\mathrm{mg}$ per day in the previous study, but mebeverine of $300 \mathrm{mg}$ was used in this study. ${ }^{2} \mathrm{I}$ wonder whether the lower dose of mebeverine may have influence on the results or not, but there was no explanation about this in the discussion. Second, I also wonder if there was any difference in the reduction of APDFS according to the subgroup of IBS patients. It has been known that mebeverine is more effective than placebo in the management of diarrhea-predominant IBS patients by diminishing stool frequency, but there is few data about the effect of $\mathrm{OB}$ on stool frequency. ${ }^{7}$ Third, the number of patients receiving rescue treatment were very high (100\% in OB and $98 \%$ in mebeverine). As mentioned in the discussion, patients were very concerned about the abnormal bowel movement activity during the treatment, therefore the rescue treatment might have been helpful for relieving their psychological stress. However, is there any possibility that this high portion of rescue therapy may affect the result by decreasing the differences of APDFS between the 2 groups?

Despite these limitations of the present study, it provides us with the important information in the management of IBS patients in Asian, because it is the first trial of $\mathrm{OB}$ in Asia. Further large-scaled studies are needed to validate the results of the present study.
1. Longstreth GF, Thompson WG, Chey WD, Houghton LA, Mearin F, Spiller RC. Functional bowel disorders. Gastroenterology 2006; 130:1480-1491.

2. Kruis W, Weinzierl M, Schlüsser P, Holl J. Comparison of the therapeutic effect of wheat bran, mebeverine and placebo in patients with 
the irritable bowel syndrome. Digestion 1986;34:196-201.

3. Maggi CA, Manzini S, Meli A. Otilonium bromide: a smooth muscle relaxant which interferes with calcium mobilization. Arch Int Pharmacodyn Ther 1983;264:305-323.

4. Poynard T, Naveau S, Mory B, Chaput JC. Meta-analysis of smooth muscle relaxants in the treatment of irritable bowel syndrome. Aliment Pharmacol Ther 1994;8:499-510.

5. Battaglia G, Morselli-Labate AM, Camarri E, et al. Otionium bromide in irritable bowel syndrome: a double-blind, placebo-controlled, 15-week study. Aliment Pharmacol Ther 1998;12:10031010.
6. Chang FY, Lu CL, Luo JC, Chen TS, Chen MJ, Chang HJ. The evaluation of otilonium bromide treatment in Asian patients with irritable bowel syndrome. J Neurogastroenterol Motil 2011;17:402410.

7. Lu CL, Chen CY, Chang FY, et al. Effect of a calcium channel blocker and antispasmodic in diarrhoea-predominant irritable bowel syndrome. J Gastroenterol Hepatol 2000;15:925-930.

\section{Conflicts of interest: None.}

